Aus der Prof. Dr. Vulpiusschen Orthopädisch-Chirurgischen Heilanstalt in Heidelberg.

\title{
Die neue Verbandbehandlung der Skoliose nach Abbott.
}

Von Prof. Dr. Oskar Vulpius.

Wenn wir uns gewiß auch darüber freuen können, in einer Zeit als Orthopäden zu arbeiten, die eine rasche, glückliche und erfolgreiche Entwicklung unserer Disziplin gebracht hat, so wird es doch wohl jedem unter uns $a b$ und $\mathrm{zu}$ so gehen wie mir:

Man wird die drückende Empfindung nicht los, daß man einer unserer wichtigsten und größten Aufgaben noch nicht in genügendem Maße gerecht werden kann, daß das Problem der Skoliosenheilung noch ungelöst vor uns steht, trotz aller wissenschaftlichen Untersuchungen und trotz mühsamer praktischer Arbeit. Und darum mußte eine Veröffentlichung von Abbott im Sommer 1911 wirken wie ein blendender Blitz. In dieser Publikation legte er kurz die Ideen dar, die ihn zu einer besonderen Art der Behandlung und zu Erfolgen geführt hatten, deren bildliche Wiedergabe Staunen erregen mußte - und zweifelndes und gar ablehnendes Kopfschütteln.

Die von Abbott angestellten Beobachtungen und Erwägungen, welche der Methode zugrunde liegen, sind in aller Kürze folgende:

Betrachten wir den Rücken eines Kindes, das sich in schlechter Schreibhaltung befindet, so sehen wir, daB die Wirbelsäule nach hinten und nach der Seite gebogen ist und daß ferner eine Verdrehung der Wirbel und mit ihnen der Rippen derart eintritt, daß der Seite der Konvexität entsprechend eine Vorwölbung der Rippen entsteht. Das Bild ist also einer Skoliose mit ihren Begleiterscheinungen durchaus analog.

Der einzige Unterschied ist der, daß die Deformität nicht fixiert ist, sondern sich sofort wieder ausgleicht, wenn das Kind aufsteht. Diese alltägliche Beobachtung suchte nun Abbott $\mathrm{zu}$ vervollständigen, indem er einen stehenden Menschen sich vornüber beugen und zugleich nach einer Seite hin biegen ließ. Auch hierdurch entsteht eine Skoliose. Beobachten wir aber die dabei eintretende Verdrehung der Wirbel und den dadurch gebildeten Rippenbuckel, so finden wir nach Abbotts Ansicht eine sogenannte falsche Skoliose, d. h. die Wirbel drehen sich nach der Seite der Konkavität. Eine konvexseitige Vorwölbung der Rippen tritt nur dann ein, wenn gleichzeitig die Schulter der konkaven Seite stark nach oben gezogen wird. Die beiden verschiedenartigen Vorgänge hat Abbott durch zahlreiche Röntgenuntersuchungen nachgewiesen.

Eine Erklärung für die Entstehung der falschen oder echten Skoliose kann Abbott zunächst nicht geben. Weil bei der vorhin erwähnten schlechten Schreibhaltung gleichzeitig die konvexseitige Schulter in die Höhe gezogen wird, während die konkavseitige Schulter herabsinkt, kommt es eben hierbei zur Bildung einer echten Skoliose oder, richtig gesagt, zu echter skoliotischer Haltung. Aus der letzteren kann die eigentliche pathologische Skoliose nur entstehen durch Umformung der Knochen, die als Folge der fehlerhaften Belastung nach dem Wolff. schen Gesetz der Transformation zustandekommt.

Abbott ging nun weiter zu dem Versuch über, an einem gesunden Menschen eine echte fixierte Skoliose zu erzeugen, wozu sich ihm ein Student der Medizin zur Verfügung stellte. In der vorhin geschilderten

1) cf. auch Anmerkung 1. 
Stellung, also bei nach vorn gebeugter und nach der linken Seite gekrümmter Wirbelsäule, wurde ein Gipsverband angelegt, während die rechte Schulter stark in die Höhe, also kopfwärts gezogen wurde. Als nach wenigen Wochen der Verband entfernt wurde, konstatierte man eine typische rechtseitige Skoliose, die mit ausgeprägtem Rippenbuckel auf dieser Seite zunächst fixiert war. Nach der Wolff schen Lehre kann nun aber auch eine Deformität dadurch beseitigt werden, daß man sie in ihr Gegenteil umstellt und dann die fehlerhafte Belastung in entgegengesetztem Sinn einwirken läßt. Der genannte Student mit seiner rechtseitigen Skoliose wurde nach der linken Seite umgekrümmt und in dieser pathologischen Stellung eingegipst. Nach wenigen Wochen fand man eine linkseitige Skoliose mit starkem linkseitigen Rippenbuckel. Durch einen dritten Verband wurde auch diese Krümmung wieder beseitigt und eine gerade Wirbelsäule erzielt.

Genau das gleiche Vorgehen wurde weiterhin von Abbott bei skoliotischen Patienten angewendet, und zwar suchte er dabei seine Theorie über die Entstehung der Brustkorbverdrehung in die Praxis zu übertragen: Diese Drehung der Wirbel und Rippen entsteht nach A b bott durch eine Vorwärtsbewegung der konkavseitigen Rippen, während die Rückwärtsdrehung der konvexen Seite nur eine sekundäre Folgeerscheinung darstellt.

Ferner verwertete Abbott bei der Verbandanlegung folgende Erwägung: Die Skoliose, die wir bei schlechter Schreibhaltung beobachten, entsteht an der kyphotisch eingestellten Wirbelsäule, also müssen wir auch die therapeutische Rückdrehung an der kyphotischen Wirbelsäule ausführen. Nur auf diese Weise können wir bei Heilung der Deformität den gleichen Weg einschlagen, dem bei ihrer Ausbildung gefolgt wurde. Es besteht also ein schroffer Gegensatz seiner Technik und der bisher üblichen:

Nicht Extension der Wirbelsäule wird angestrebt, sondern ihre Zusammendrückung in nach hinten gerichtetem Bogen; nicht Druck auf den konvexseitigen hinteren Rippenbuckel wird ausgeübt, sondern starke Entlastung wird herbeigeführt auf der konkavseitigen hinteren Rückenfläche. Das Problem, das durch die Technik des therapeutisch wirksamen Skoliosenverbandes gelöst werden muß, ist also folgendes:

Es muß die Wirbelsäule im Gipsverband fixiert werden in Kyphose und in Ueberkorrektur der seitlichen Biegung. Es muß die konkavseitige hintere Rückenfläche frei bleiben und nach hinten gedrängt werden. Auf den konvexseitigen Rippenbuckel darf kein Druck ausgeübt werden, denn hierdurch würde nur eine Streckung der Wirbelsäule erzeugt werden. Die Streckstellung der Wirbelsäule aber würde die seitliche Umkrïmmung zum mindesten erschweren oder unmöglich machen, weil in dieser Stellung nach Lovetts Untersuchungen eine Verhakung der Brustwirbel untereinander eintritt.

Als ich diese erste Arbeit von Abbott studiert hatte, welche die kurz skizzierten Erwägungen und Probleme noch nicht ganz klar darlegte und die technische Lösung nicht beschrieb, versuchte ich das Verfahren zunächst am sitzenden Patienten. Die Sitzfläche wurde nach vorn abfallend gestaltet, um die Verbandanlegung bis zu den Hüftgelenken herunter zu erleichtern. Der Patient wurde aufgefordert, eine möglichst kyphotische Haltung einzunehmen und die seitliche Beugung nach Kräften zu korrigieren. Der Arm der konvexen Seite wurde durch einen Bindenzügel nach vorn und unten gezogen, der Arm der konkaven Seite mit Hilfe eines zweiten Zügels senkrecht in die Höhe und über den Kopf weg.nach der Seite der ursprünglichen Konvexität hin. Entsprechend der konkavseitigen Rückenfläche wurde in den etwas unterpolsterten Verband ein größeres Fenster geschnitten. Wir konnten uns auf diese Weise an einer Reihe von Patienten überzeugen, da $B$ in der Tat eine Besserung eintrat. Indessen gelang es uns nicht, Erfolge zu erzielen, die mit den von $\mathrm{A}$ b bott abgebildeten konkurrieren konnten.

Nun erschien im Frühjahr 1912 eine zweite Arbeit von Abbott, die über glänzende Resultate berichtete und zugleich technische Anleitungen enthielt. Es zeigte sich, daß Abbott einen besonderen Lagerungstisch verwendete, auf dem die Patienten mit Hilfe von Bindenzügeln in der gewünschten Weise gelagert wurden.

Ich lieB unir daraufhin einen solchen Originaltisch aus der Abbott. schen Werkstätte kommen, konnte aber nach dessen Eintreffen mich mit der Anwendungsweise trotz freundlicher Auskunft des Erfinders nicht recht vertraut machen. So entschloß ich mich kurzerhand, das orthopädische Wunder an Ort und Stelle kennen zu lernen, und folgte im Herbst 1912 einer hebenswürdigen Einladung Abbotts nach der Stätte seiner Tätigkeit in "Portland, wo ich"mit größter Gastfreundschaft aufgenommen wurde. Es wurde mir aufs entgegenkonmendste eine Reihe von Patienten demonstriert, es wurde mir die Verbandanlegung gezeigt und sogar die Möglichkeit gegeben, unter Abbotts Leitung solche Verbände auszufiuhren. Nach meiner Rückkehr aus Amerika machte ich mich, angefeuert durch die dort gewonnenen Eindrücke, mit großem Eifer daran, die Methode in ihren technischen Einzelheiten durchiustudieren, und habe jetzt etwa ein Viertelhundert skoliosen in Verbänden gehen.

Da ich mich von der Wirksan keit der Methode nicht nur, sondern auch von deren zahl reichen Schwierig keiten überzeugt habe, so halte icl es für meine Pflicht. durch eingehende Beschreibung der Technik möglichst schnell meine Kol legen in den Stand zu setzen, das Verfahren aufzuneh inen.

Ohne den Abbottschen Lagerungstisch ist die Anlegung eines korrekten Verbandes nicht möglich.

Dieser Apparat (Fig. 1 u. 2) besteht aus einem system von rechtwinklig miteinander verbundenen Röhren, die drei übereinander liegende Horizontalrahmen bilden. Die sechs Längsstangen lassen sich um ihre Achse drehen und durch ein Zahnrad in jeder gewünschten Stellung fixieren. Innerlıalb des oberen Rahmens wird eine Art Hängeinatte angebracht, die sich an ihrem Fußende beliebig stark anspannen läßt. Die Seiten dieser Hängəmatte sind ungleich lang; wird also die eine Längsseite angespannt, so ble bt aie entgegengesetzte Seite schlaff. Hierdurch wird ein einseitiges Heruntersinken der Rückenfläche ermöglicht. Außerdem trägt dieser obere Rahmen zwei Quergurte, von denen der eine den Nacken des Patienten, der andere das Becken stützen soll. Zwischen diesen beiden Gurten kann sich dann die Wirbelsäule kyphotisch nach unten senken. Die kyphotische Haltung wird dadurch noch verstärkt, daß die Beine eleviert werden, und zwar ge schieht dies mit Hilfe eines Flaschenzuges, der in entsprechender Höhe über dem oberen Horizontalrahmen angebracht ist. Die Stanyen, die diesen Flaschenzug tragen, stehen endlich in Verbindung mit einem vierten obersten Horizontalrahınel, dessen Seitenteile ebenfalls in der vorhin geschilderten Weise um ihre Achse duehbar und durch Zahnrad feststellbar sind. Die acht demnach vorhandenen drehbaren Rahmen-

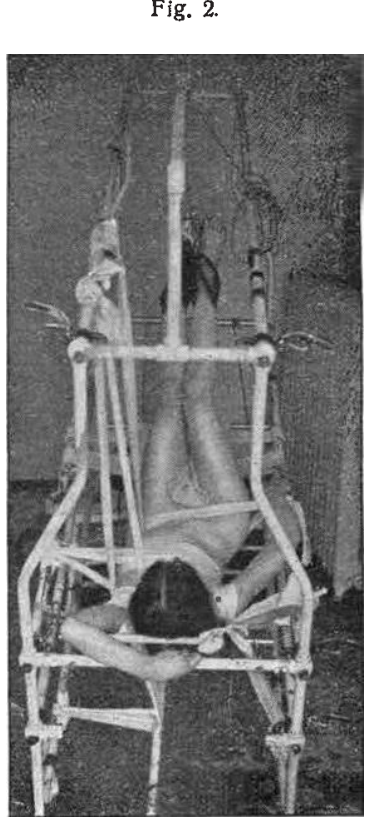

schenkel sind mit einer Art verschiebbarer Klammervorrichtung velsehen, die dazu dient, die anzubringenden Bindenzügel festzuhalten. Endlich wäre noch zu erwähnen, daß der unterste Rahmen eine mittlere, seitwärts verschiebliche Längsstange aufweist. ${ }^{1}$ )

Der Patient wird in folgender Weise zum Eingipsen vorbereitet:

Die gut gereinigte Haut wird tüchtig eingepudert, der Rumpf mit zwei oder drei Trikotschläuchen bekleidet. Zwischen die verschiedenen Trikotschläuche kommt eine Anzahl dicker Filzpolster, welche die gefährdeten Stellen des Beckens, der Brust und der Achs@lhöhlen vor Druck schützen. Auch die konvexseitige seitliche Rumpffläche wird gepolstert. Ein weiteres Polster wird in die konkavseitige Einsenkung der

1) Der Abbottsche Originalapparat kann von Herrn Franz Bingler, Bandagisten in Heidelberg, bezogen werden. 
Rückenfläche eingelegt; dieses wird später, nachdem, ein Fenster in den Verband geschnitten worden ist, wieder entfernt. Das Polster dient nur dazu, um im Verbandfenster für die sich vordrängenden Rippen Platz zu machen. Nun wird der Patient in der vorhin beschriebenen Weise in möglichst kyphotischer Stellung in die Hängematte gelagert. Die Beine werden stark nach oben gezogen. Es folgt die Anlegung der redressierenden Bindenzügel, die zunächst etwas kompliziert erscheint, bei einiger Uebung aber durchaus selbstverständlich wird. Wir nehmen bei der Beschreibung den Fall einer rechtseitigen Skoliose an. Der Patient liegt auf der Hängematte so, daß die linke Rückenhälfte auf deren erschlaffter linker Seite heruntersinkt. Die linke Schulter wird dadurch in die Höhe gezogen, daß der linke Arm unter den Kopf zu liegen kommt. Der rechte Arm ruht auf der oberen rechten Längsstange. Fine Binde umgreift die linke Achselhöhle von vorn, die Bindenenden gehen unter der Hängematte nach der rechten oberen Längsstange. Eine zweite Binde umzieht die linke Schulter von hinten und geht hinauf zum linken obersten Längsstab. Eine dreiteilige Binde umkreist das Becken von der linken Seite her, zwei ihrer Enden werden an der rechten oberen Längsstange befestigt. Das dritte Ende dieser Binde geht von der Wirbelsäule nach links und oben und endigt an der obersten linken Längsstange. Die drei bisher beschriebenen Bindenzügel haben den Zweck, Schultergürtel und Becken nach rechts zu fixieren, die linke Schulter und die linke Beckenseite nach oben zu heben. Eine vierte Binde umfaßt die rechte Achselhöhle von oben her und wird an untersten rechten Längsstab befestigt. Sie hat die Aufgabe, die rechte Schulter abwärts zu ziehen. Eine weitere dreiteilige Binde zieht über den Scheitel der rechts konvexen Brustskoliose weg, zwei ihrer Enden werden am oberen oder besser am mittleren linken Längsstab festgebunden, das dritte Bindenende umzieht die rechte Seite des Brustkorbes, geht über den vorderen Rippenbuckel nach links und unten, wird um den Mittellängsstab des untersten Horizontalrahmens geschlungen und endigt am linken unteren äußeren Längsstab. Dieser Zügel hat die Aufgabe, die Wirbelsäule umzukrümmen und gleichzeitig die Rückdrehung des Thorax zu erzeugen.

Alle beschriebenen Zïgel werden nacheinander durch entsprechendes Drehen der Längsstangen angespannt, bis die gewünschte Korrekturstellung erzeugt ist, worauf ein genügend dicker Gipsverband angelegt wird. Nach dem Erstarren des Verbandes wird der Patient losgeschnitten und aut einem Tisch gelagert. Es folgt das Einschneiden einer Reihe von Fenstern. Ein größeres ovales Fenster entspricht der konkavseitigen Rückenhälfte, durch das die vorhin erwähnten Filzpolster entfernt werden können. Ferner werden drei schlitzfölmige Fenster angebracht, je eines in der vorderen und hinteren Achsellinie rechts, ein drittes Fenster auf der Vorderfläche
Fig. 3.

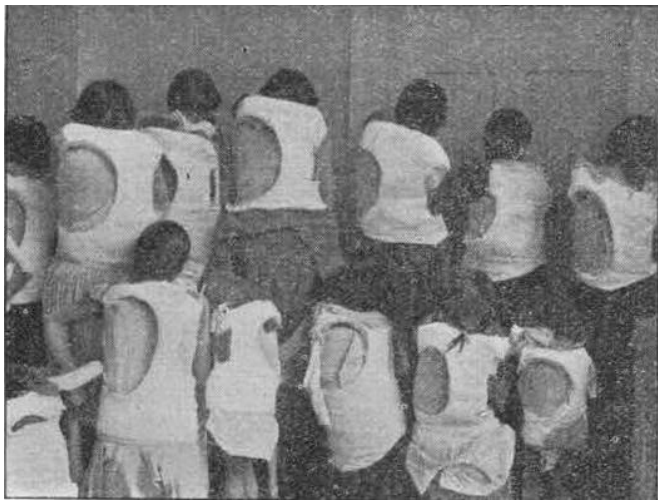
etwa in deren Mitte. Größe und gegenseitige Lagebeziehung der Fenster richten sich nach den speziellen Verhältnissen des Einzelfalles (Fig. 3 u. 4). Der Patient kann längstens nach einigen Tagen das Bett vellassen und tritt nun in die $\mathrm{Pe}$ riode der Nachbehandlung ein.

Von der sorgfältigen Durchführung dieser Nachbehandlung hängt sehr viel, um nicht zu sagen alles ab. Es werden, soweit der Zustand es zuläßt, leichte gymnastische Uebungen vorgenommen, um einer allzu großen Schwächung der Rückenmuskulatur vorzubeugen, ferner, worauf ungemein viel an- kommt, täglich mehrnials wiederholte Atmungsgymnastik. um die Fntfaltung des Brustkorbes zu beschleunigen. Vor allen Dingen werden Polster eingeschoben, welche die beginnende $\mathrm{Tm}$ -

Fig. 4.

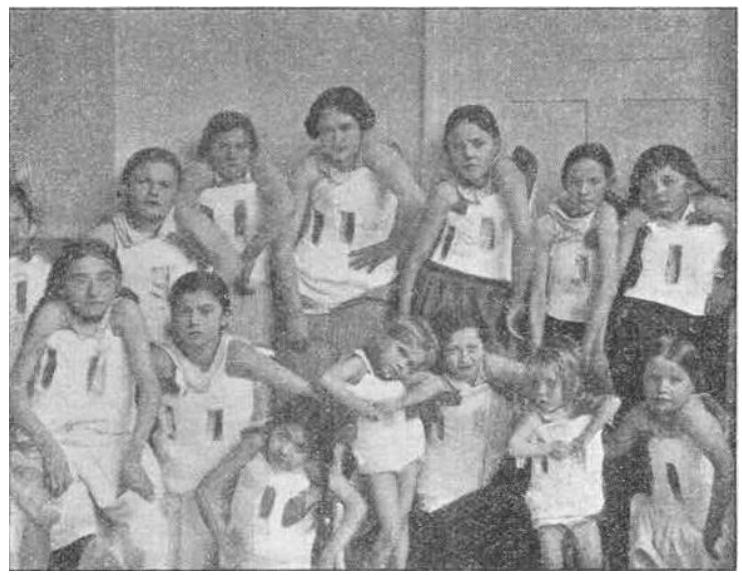

krümnıung der Wirbelsäule fördern sollen. Zı diesem Zwecke werden, sobald der im Gipsverband entstehende Hohlraum es zuläßt, mehr oder weniger dicke Filzpolster unter den Verband geschoben, und zwar einunal zwischen den beiden schlitzförmigen Fenstern der rechten Seite, ferner zwischen dem Schlitz auf der Vorderfläche des Verbandes und dem großen ovalen Fenster. Das rechtseitige Polster hat den Zweck, die seitliche Ueberkorrektur herbeizuführen. das Vorderpolster soll die Rippen nach hinten drängen, also die Rückdrehung erzeugen. Je nach dem Verlauf werden die Verbände in Zwischenräumen von einigen Wochen oder Monaten gewechselt. Die Dauer der Verbandperiode richtet sich nach dem einzelnen Fall und dem Fortschreiten der Heilung. Sie kann einen Zeitraum umfassen, der zwischen wenigen Wochen und sechs bis acht Monaten schwankt. Während dieser Zeit ist dem Skelett die Möglichkeit gegeben, sich umzuformen und in Korrekturstellung zu fixierell. Nach definitiver Entferıung des Gipsverbandes beginnt die gymuastische Nachbehandlung in bekannter Weise. Es empfiehlt sich, das Resultat während der ersten Zeit wenigstens durch ein Stützkorsett festzuhalten, dis unter Uniständen in Ueberkorrekturstellung des Rumpfes angefertigt wird.

Es ergibt sich aus dem Gesagten wohl, daß die Behandlung nicht ganz einfach und ziemlicll langwierig ist und daß sie außerdem unzweifelhaft mit Unbequemlichkeiten für den Patienten verbunden ist obwohl die Kinder sich in gut sitzenden Verbänden garnicht unglïcklich fühlen. So ist die Frage von vornherein berechtigt und notwendig, ob die Erfolge in richtigen Verhältnis zu den genannten Nachteilen stehen.

Wir befindelı uns im Anfang der Entwicklung einer neuen Methode und müssen darum mit unseren Urteil gewiß noch vorsichtig sein. Wenn ich über Erfolge der Methode berichte, so geschieht dies einmal auf Grund nieiner in Amerika gewonnenen Eindrücke, ferner auf Grund der Erfahrungerr, die ich selber bisher zu sammeln Gelegenheit gehabt habe. Ich habe mich sowohl bei den amerikanischen als auch bei meinen eigenen Patienten stets davon überzeugen können, daß durch die Art der A b bot tschen Lagerung die Umkrümmung erzielt oder wenigstens erleichtert wird. Wenn wir nach Erhärtung des Verbandes das große ovale Fenster ausgeschnitten haben, so konstatieren wir sofort die Verschiebung des Thorax nach der Seite der Konkavität, und wir sehen weiter zwischen hinterem Verbandrand und Körper einen bisweilen recht großen Hohlraum, dessen Tiefe etwa der Dicke des eingelegten Polsters entspricht (Fig. 5). Aber schon nach wenigen Stunden können wir gewöhnlich beobachten, daß dieser Hohlraum sich velkleinert, nach einigen Tagen völlig verschwindet, ja es kann die Umformung des Brustkorbes so weit gehen, daß die Rippen sich gegen den Rand des Fensters stark anstemmen und daß hier durch weiteres Ausschneiden oder durch Unterpolstern ein Decubitus verhütet werden muß (Fig. 6). Je mehr wir 
nachpolstern, je eifriger die Atmungsübungen durchgeführt werden, desto stärker wird die Korrektur und das Hervorquellen del' konkavseitigen Rüickenfläche rach hinten.

Fig. 5 .

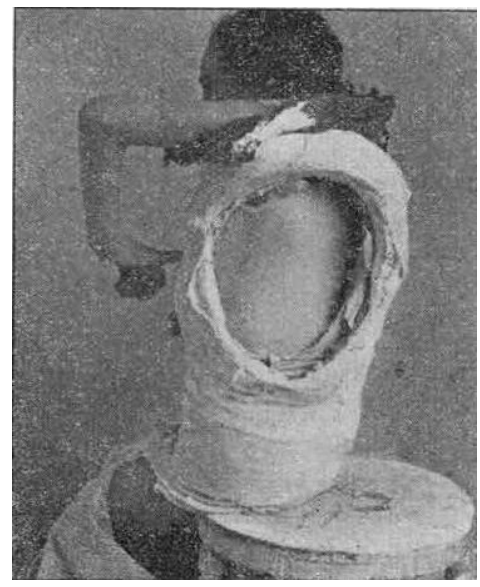

Der palpierende Finger kann von hier aus die Abflachung des skoliotischen Bogens verfolgen und unter Umständen sogar dessen Ueberkorrektur konstatieren. Bein Verbandwechsel ist die fortschreitende Heilung festzustellen, doch darf

Fig. 7.

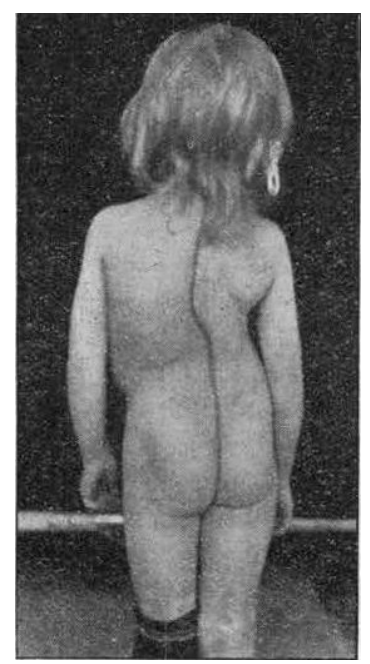

F. U., 6 Jahre, vor Anlegung des verbandes.
Fig. 6

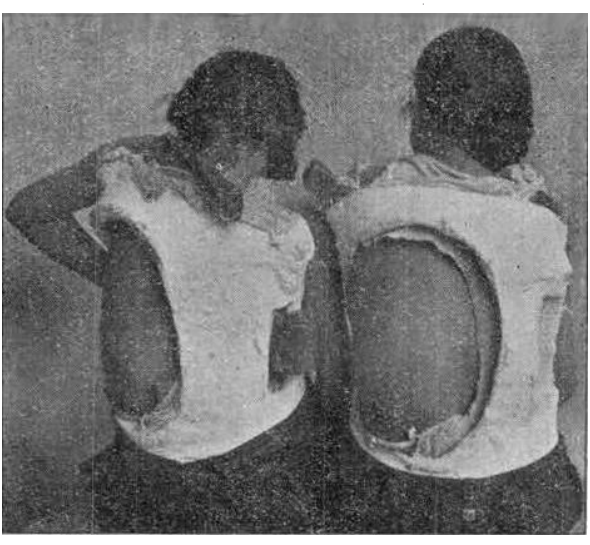

Neben dieser Gefahr des Rezidivs bestehen auch andere Crefahren der Behandlung, die man nur durch C'ebung zu vermeiden lernt. Die größte Gefahr ist natürlich der Decubitus, der sowohl in der Achselhöhle als auch auf der Höhe des Rippenbuckels oder am Rand der Fenster eintreten kann. Eine exakte Polsiterung ist also unerläßlich, außerdenı muß nach Möglichkeit das Eindringen von Fremdkörpern in den Verband verhütet werden. Auch ein schädlicher Druck auf die Nerven der Achselhöhle ist sorgfältig zu vermeiden, wenn wir nicht unangenehme Ueberraschungen erleben wollen.

Es ist heute noch unmöglich. Zahl und Guite der Dauererfolge zu berechnen, weil die Beobachtungszeit hierzu viel zu kurz ist. Soviel aber kann jetzt schon gesagt werden, daß mit Hilfe dieses Verfahrens Umkr ü mmungen. der Wirbelsäule erreicht werden können, die bis dahin vollständig unmöglich waren. Und zwar gilt dies sowohl fïr die seitliche Verbiegung der Wirbelsäule als für den Rippenbuckel, dessen Abflachung im Laufe weniger Monate in der Tat erstzunlich ist.

Um dies in einwundfreier Form zu veranschaulichen, dazn ist die Photographie selbstverständlich unzureichend,

Fig. 9.

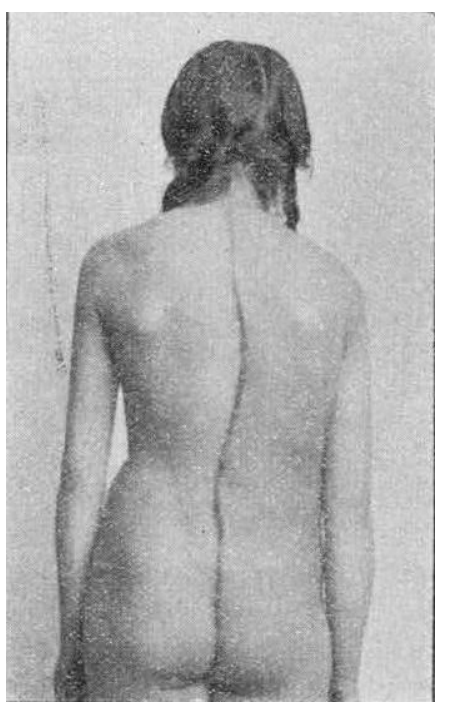

M. L., 11 Jahre, vor Beginn der Behandlung.
Fig. 10.

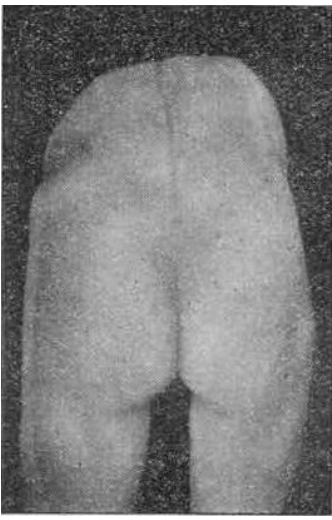

Dieselbe vor Beginn der Behandlung.

man sich durch diesen an sich erfreulichen Befund nicht dazu verleiten lassen, friihzeitig die Verbandbehandlung aufzugeben. Ein Rïckfall würde die unvermeidliche Folge der verfrühten Freude sein!

Fig. 11.

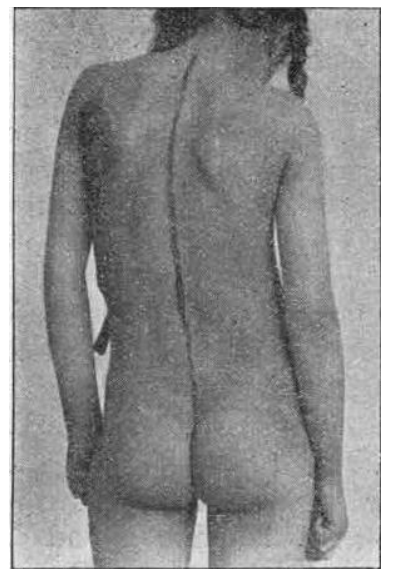

Dieselbe nach 3 Monaten (2 Verbände).
Fig. 12 .

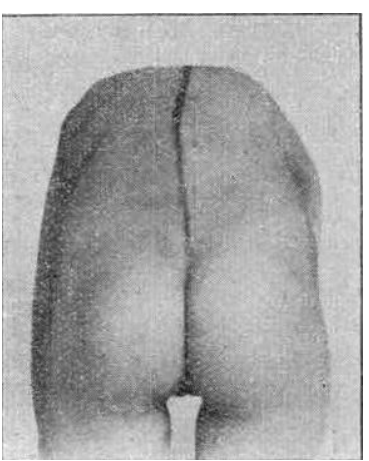

Dieselbe nach 3 Monaten (2Verbände).

auch: wenn sie unter Anwendung aller Vorsichtsmaßregeln in aufrechter und vorgebeugter Stellung des Patienten hergestellt wird. Für Dritte überzengend kann eigentlich nur der Gips-

Fig. 13.

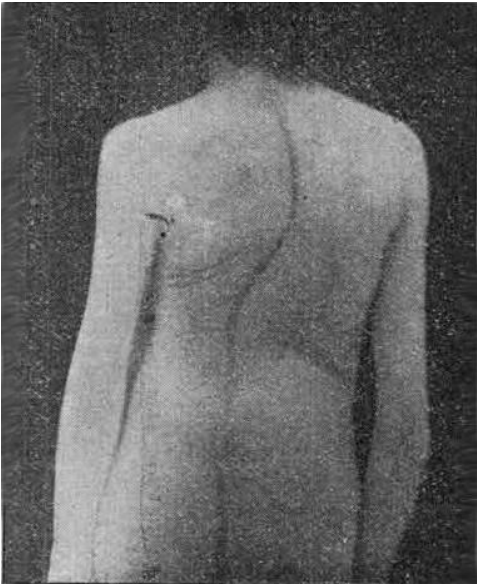

P. Z., 14 Jahre, vor der Behandlung.
Fig. 14.

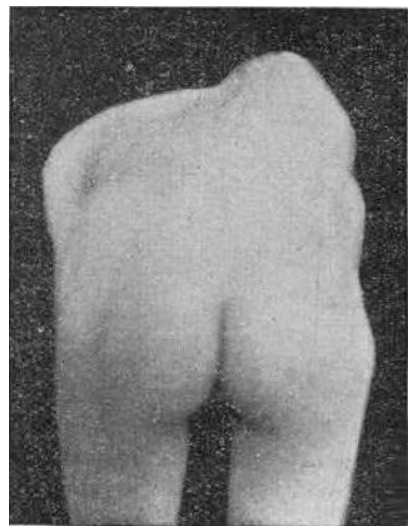

Dieselbe vor Beginn der Behandlung. 
abguß wirken. Ich habe mich darum der Mühe unterzogen, solche Abgüsse von allen meinen Patienten sowohl in Bauchlage wie in vorgebeugter Stellung anzufertigen, ehe die Behandlung begonnen wurde, und während ihres Verlaufs. Mit Hilfe dieser freilich zeitraubenden Methode, die, wenn auch nicht ein ganz untrïgliches, so doch ein verhältnismäßig zuverlässiges Beweismittel darstellt, habe ich meine Ueberzeugung von dem Wert der A b bottschen Behandlung gefestigt

Fig. 15:

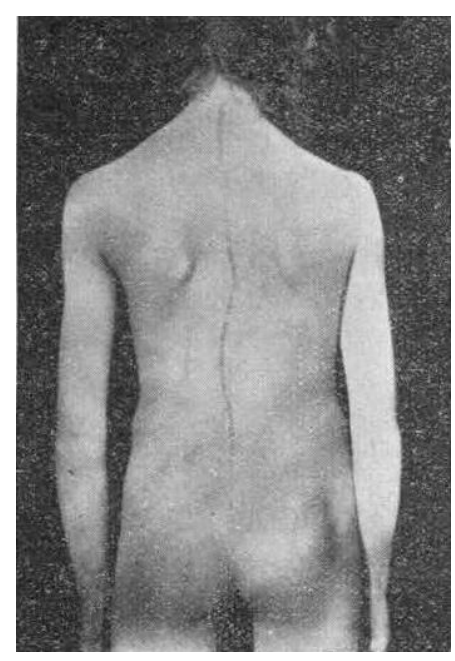

Dieselbe nach 3 Monaten (2 Verbände). und die Erfolge Kollegen vorlegen können. Daneben wird freilich auch die Röntgen platte

Fig. 16.

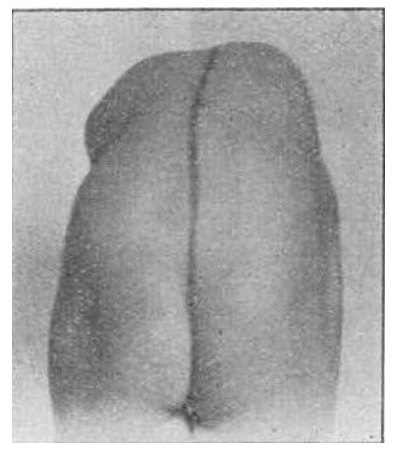

Dieselbe nach 3 Monaten (2 Verbände).

nit einem noch höheren Maße von Beweiskraft dartun können und müssen, was die A b bott-

sche Behandlung $\mathrm{zu}$ leisten vermag. A b bott selber hat bereits mehrere Röntgenbilder veröffentlicht, die mehr als nur $\mathrm{Zu}^{-}$ friedenstellendes erkennen lassen.

Zum Schluß möchte ich einen nahe liegenden Einwand kurz streifen, nämlich den, ob nicht die Korrektur der Hauptkrümmung eine Verstärkung der Nebenkrümmungen nach sich ziehen müsse. A bbott selber legt auf diese sekundären Krümmungen keinen allzu großen Wert und bekämpft diese durch nachträgliche Korrekturen an seinem Verband. Besteht im Falle einer rechtseitigen Brustkrümmung die linkseitige Gegenkrümnuung des Lendenteiles, so wird der Verband rechts unten seitlich ausgeschnitten, das Becken durch linkseitige Einschiebung von Polstern nach rechts gedrängt und unter starkem Herabziehen der rechtseitigen Beckenhälfte wieder in den ergänzten Rumpfverband eingezogen. In analoger Weise geht Abbott gegen die obere Gegenkrümnung späterhin vor. In der Hauptsache aber glaubt er diese Gegenkrümmungen, insoweit sie etwa durch den Verband verstärkt wurden, durch geeignete Nachbehandlung wieder beseitigen zu können. Tch selber lasse nachts eine Extension an dem der lumbalen Konkavität entsprechenden Bein wirken und erhöhe tagsiiber den Stiefel des anderen Beines. Wenn ich der ersten Publikation in Deutschland über dieses äußerst aktuelle Thema einige Abbildungen von behandelten Patienten beigebe, so soll damit kein abschließendes Urteil über die Erfolge der Behandlung herbeigeführt werden. Die Bilder sollen vielmehr nur dazu dienen, Zweifler zu eigenen Versuchen $\mathrm{zu}$ ermutigen, in der gleichen Weise, wie derartige frappierende Resultate mich selber immer wieder anspornen und den Glauben in mir festigen, da $\beta$ die neue Methode in der Tat neue und unerwartete Perspektiven für die Skoliosenheilung erschließt. 